\title{
Content Based Image Retrieval Using Transform Domain Features and Algorithms
}

\author{
Shaik. Rahamtula, T. Jaya
}

\begin{abstract}
In this paper, Content Based Image Retrieval using Transform domain features and algorithms has been implemented. The image can be decomposed by Discrete Wavelet Transform (DWT) to extract the features based on DC coefficients. Each sub-image is calculated by mean, variance and standard deviation to get more efficient recognition. The database image also applied in the domain of Stationary Wavelet Transform (SWT) and Integer Wavelet Transform (IWT) by using different distance measures. The proposed algorithm is the combination of DWT, SWT and IWT has been implemented using COREL database. This proposed method has more efficient recognition and less computational time over existing methods
\end{abstract}

Index Terms: Content Based Image Retrieval, Discrete Wavelet Transform, Stationary Wavelet Transform and Integer Wavelet Transform.

\section{INTRODUCTION}

From the past years researchers collects source in digital form using digital devices such as camera, scanner [1]. For the efficient process of retrieval and querying digital image from huge image database CBIR (Content Based Image Retrieval) is the best method [2]. In CBIR image retrieval is based on the contents of the image [3]. The content of the image can collect as features of the image and depends on the similarity of the collected features the similar image can retrieve from huge digital image database. The CBIR is the process of searching and retrieving images from a database on the basis of features those are extracted from the image themselves [2] [5]. The block diagram of CBIR system is shown in figure 1.

CBIR algorithm is used to overcome the drawbacks of text-based retrieval system. The TBIR (Text Based Image Retrieval) is high reliable technique. Speed of TBIR decrease if image database size reduces and vice-versa. [1] [4] [5]. The CBIR researchers worked hard to find reliable and fast search methods [6].

Image contents are classified as (a) spatial (b) semantic (c) low-level contents [2]. Position of the object in an image consider as content in the spatial domain. The semantic content gives the meaning of the image. The low-level content represents the colour, shape and texture features.

In this paper WANG database has been used. It has 1000 images from 10 different classes. The database is available in internet. The following link helpful to download the database

Wang.ist.psu.edu/docs/related

Revised Manuscript Received on October 15, 2019.

Shaik. Rahamtula, Research scholar, Department of Electronics and Communication Engineering, VELS Institute of Science Technology and Advanced Studies, Pallavaram, Chennai, Tamilnadu, India.

Dr. T. Jaya, Assistant Professor, Department of Electronics and Communication Engineering, VELS Institute of Science Technology and Advanced Studies, Pallavaram, Chennai, Tamilnadu, India.
The features are extracts using colour-autocorrelogram, colour moments, HSV, DWT and SWT. The extracted features are stored as a feature database. Consider an image as query image from database and apply the same feature extraction techniques. Finally compare the features of query image with the features of each of the database images using different distance metrics. The minimum distance is the top retrieved image.

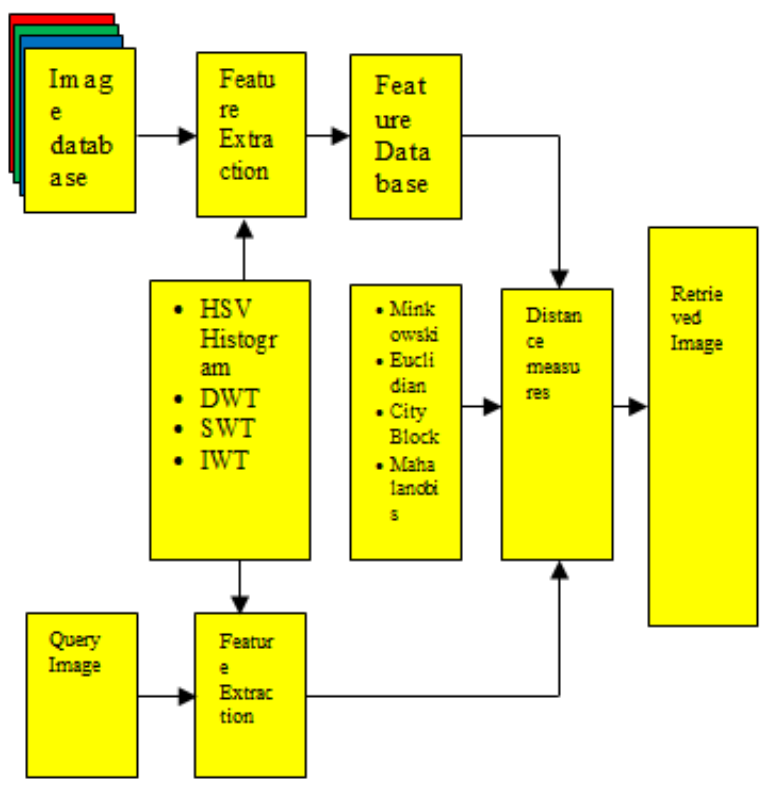

Figure 1 Architecture of CBIR system

\section{FEATURE EXTRACTION TECHNIQUES}

Feature extraction is directly affects the retrieval rate in CBIR so the feature extraction task is very important in CBIR system [2]. CBIR uses different techniques. Some of they are

A. Color moments.

B. HSV histogram features.

C. DWT.

D. SWT

E. IWT

Feature extraction is essential CBIR [1]. Image contents effects the accuracy and performance of feature extraction based image retrieval [1].

\section{A. Colour moments}

The colour indexing process is mainly depends on colour moments. The colour indexing is used in CBIR to compare the similarity of two images such as one from query and other one from database.

Published By:

Blue Eyes Intelligence Engineering

\& Sciences Publication 
a) Mean b) Standard deviation c) Skewness are color moments [7]. They can understand one by one as follows.

\section{Mean or average:-}

Mean defines the average colour present in the image. The mean of the image can be calculated as

$$
\left.E_{i}=\sum_{j=1}^{N} \frac{1}{N} p_{i j} \text { (or) Mean } E_{i}\right)=\frac{1}{N} \sum_{j=1}^{N} I_{i j}
$$

Where $\mathrm{N}=256 \mathrm{X} 385, \mathrm{Pij}$ is $\mathrm{jth}$ pixel value and ith colour channel [3][8].

Mean calculation involves two steps they are,

Step 1: Get R, G and B color components from inserted color image individually.

Step 2: Calculate the mean of the every component.

\section{Standard deviation:-}

It can be calculate as

Standard deviation $\left.\sigma_{i}\right)=\sqrt{\left(\frac{1}{N} \sum_{j=1}^{N}\left(p_{i j}-E_{i}\right)^{2}\right)}$

The process of $\sigma_{i}$ involves following steps

Step 1: Get R, G and B color components from inserted color image individually.

Step 2: Calculate the mean of the every component.

Step 3: Compute the variance of the every component.

Step 4: Finally compute the $\sigma_{i}$ using above equation.

\section{Skewness:-}

It can be calculate as [3][8].

$$
\text { Skewness } \left.\left(\mathrm{S}_{\mathrm{i}}\right)=\sqrt[3]{\left(\frac{1}{N}\right.} \sum_{j=1}^{N}\left(p_{i j}-E_{i}\right)^{3}\right)
$$

\section{B. HSV Histogram Quantization:-}

Figure 2 shows HSV plane. It reveals the dimensions of color components in HSV cone [2] [6].

HSV histogram features can be obtained as

Step 1: Converting the input color image into HSV colour space [9].

Step 2: Computing the quantization [9] of HSV colour model.

Step 3: Histogram computation of each quantized image.

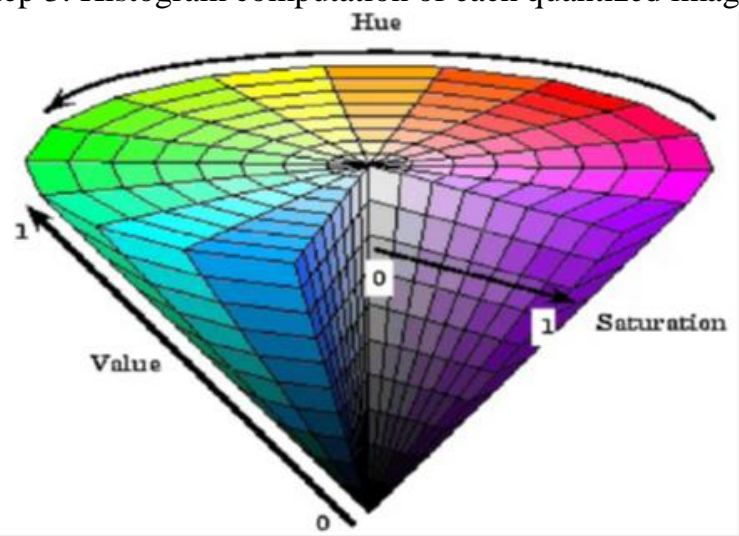

Figure 2 Planes of HSV colour space

RGB input image can convert into HSV colour space by using the following equations

$$
\begin{aligned}
H & =\cos ^{-1} \frac{\frac{1}{2}[(R-G)+(R-B)]}{\sqrt{(R-G)^{2}+(R-B)(G-B)}} \\
S & =1-\frac{3[\min (R, G, B)]}{R+G+B} \\
V & =\left[\frac{R+G+B}{3}\right]
\end{aligned}
$$

\section{Discrete Wavelet Transform:-}

The time domain signal can be converts into time and frequency domain signal by using the Discrete Wavelet Transform (DWT). Non-stationary signals processing is possible with the help of DWT only. In wavelet transform we get both time and frequency information. In Fourier transform we lose the time information after transform is performed . DWT depends on sub-band coding. Wavelet transform can be computed in high speed with DWT. DWT used to reduce resources required. It also used to reduce the time required for computation .

The figure 3 shows the 4-level DWT sub-band coding on the digital image.

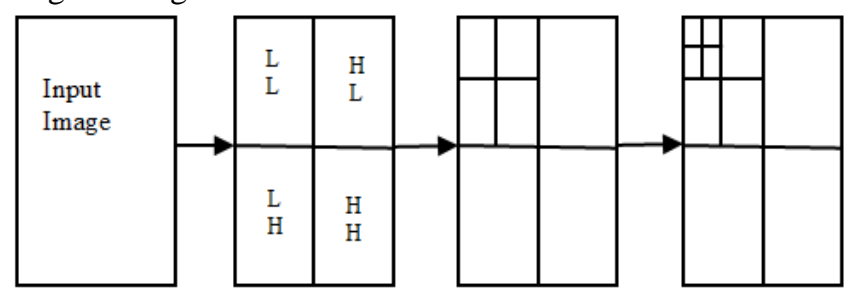

Figure 3 DWT 4-levels decomposition

This process repeated up to four levels [1] .

\section{Stationary Wavelet Transform (SWT):-}

Before introducing the SWT the researchers widely used DWT for feature extraction. The DWT is not applicable for time variant property. To overcome the limitations of DWT a multi-layer stationary wavelet transform (SWT) is used . The SWT provides excellent solutions in the applications of image processing. SWT is redundant, linear and shift invariant . The SWT is also referred as UWT, IWT \& RWT.

SWT is similar as DWT except scaling function. The four outputs of SWT sizes are as same as the original image [20]. The 2-dimentional wavelet decomposition obtained by 1-dimentional SWT along the rows of the image and then decomposed along the columns [10].

This decomposition gives four decomposed sub-band images as shown in figure4 [10]. The sizes of sub-band images and input image are similar [10]. Most of the information of the given image is available in the LL subband. $\mathrm{HH}$ sub-band contains the detailed information regarding the edges.

The figure 4 shows the image decomposition using SWT. 


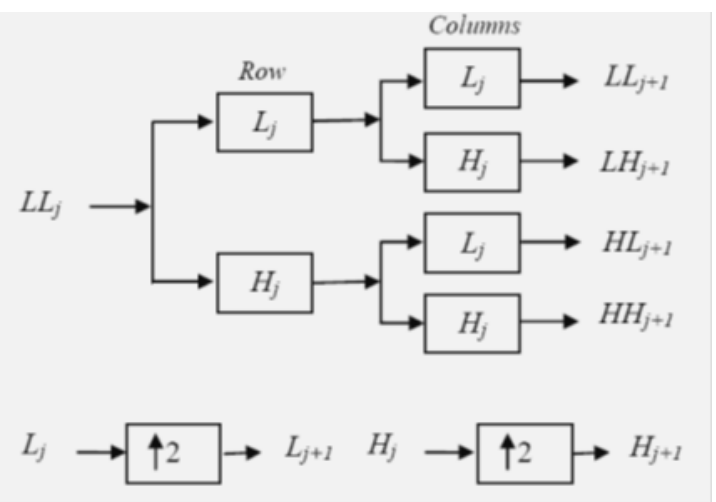

Figure 4 Image decomposition using SWT

\section{E. Integer Wavelet Transform (IWT):-}

In DWT method the wavelets are directly sampled. It suitable for CBIR to represent image features effectively. But the DWT produce the result in floating point . It creates reconstruction problem. In 1998 Sweldens introduced a method which can nicely overcome the drawback of DWT called Integer Wavelet Transform (IWT). Lifting scheme introduced to construct IWT . Lifting scheme is one of the structural methods, in which all the constructions are derived in spatial domain. It is a novel approach for constructing second generation wavelet. It has convenient construction, in-place calculation, lower computational complexity and simple inverse transform. IWT is reversibility i.e. image can be reconstructed without loss of original image information, because all coefficients are integers and can be stored without rounding off errors .

The figure 5 shows the basic structure of lifting scheme implemented in two dimensions.

The basic lifting scheme can be easily understand with the following three concepts.

\section{(i). Split (S) \\ (ii). Predict (P) \\ (iii). Update (U)}

(i). Split (S):- It is also known as Lazy Wavelet Transform, because in splitting concept we not performing any mathematical operation, we just splitting the input samples into even and odd parts .

The even samples and odd samples can be represented as

$$
\begin{aligned}
& x_{e}(n)=x(2 n) \\
& x_{0}(n)=x(2 n+1)
\end{aligned}
$$

Each group (or) frame (or) part contains one half samples of the original signal.

(ii). Predict $(\mathrm{P})$ :- Few researchers identify this as dual lifting [22]. Here we predict odd samples by using even samples. The wavelet coefficient can be obtained by subtracting the predicted even value from the actual odd value [23].

$$
\mathrm{H}=\mathrm{x}_{\mathrm{o}}(\mathrm{n})-\mathrm{P}\left[\mathrm{x}_{\mathrm{e}}(\mathrm{n})\right]
$$

Let $\mathrm{P}$ be the predict operator

(iii). Update (U):- It is also known as primal lifting.It follows predict step. Update happened by sum of input even samples and updated odd sample. Then we get scaling coefficients. Update denoted by a letter U.

$$
\begin{aligned}
& \text { coefficients. Update denoted by a letter } U \text {. } \\
& L=x_{e}(n)+U[H]
\end{aligned}
$$

The IIWT can be obtained as

$\mathrm{x}_{\mathrm{e}}(\mathrm{n})=\mathrm{L}-\mathrm{U}(\mathrm{H})$

$\mathrm{x}_{\mathrm{o}}(\mathrm{n})=\mathrm{H}+\mathrm{P}\left[\mathrm{x}_{\mathrm{e}}(\mathrm{n})\right]$

$x(n)=\operatorname{Merge}\left(x_{e}(n), x_{0}(n)\right)$

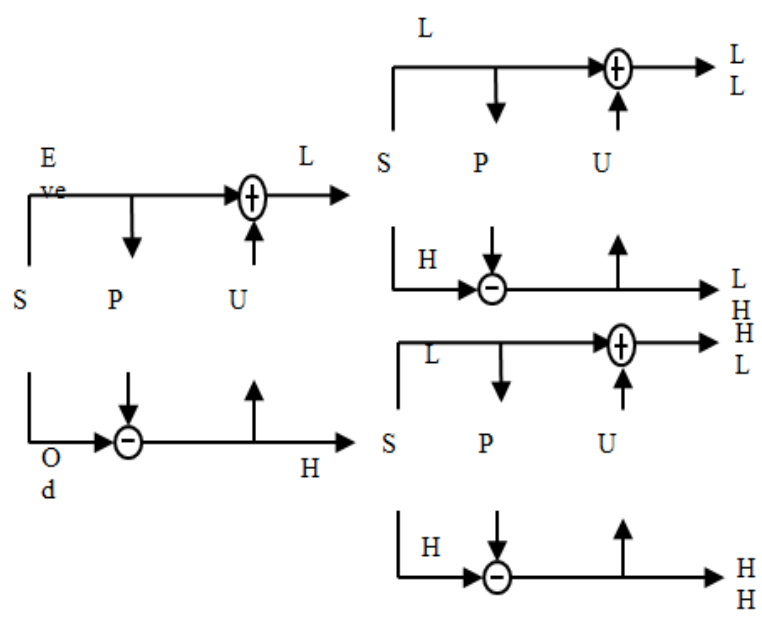

Figure 5 2D Lifting Scheme Implementation

\section{PROPOSED METHOD}

Step 1:- Set the Path of database.

Step 2:- Get gray scale image from RGB image. Resize the image for [256 256] because the selected database images are in size of $256 * 384$ or $384 * 256$.

Step 3:- To perform DWT operation consider the following steps.

Step a:- On complete gray image apply DWT with Haar filter. It produce Low-Low sub-band.

Step b:- Generate the Mean (M) and Standard Deviation (Sd) for Low-Low Sub-band.

Step c:- Above computed Feature vectors form as single vector i.e $\mathrm{fv} 1=[\mathrm{m} 0 \mathrm{std} 0]$ and consider them as features for image retrieval.

Step 4:- To perform SWT operation consider the following steps.

Step a:- On complete gray image apply Stationary Wavelet Transform (SWT) using db6 filter. It produce Approximation sub-band (ca).

Step b:- Separate the 4 sub layers of 4 individual subbands of ca, ch, cv and cd. The 4 sub layers of ca are let be named as A1, A2, A3 and A4.

Step c:- Generate the Mean (M) and Standard Deviation (Sd) for all 4 sub layers of approximation Sub-band ca.

Step d:- Above computed feature vectors form as single vector fv2 $=\left[\begin{array}{llllllll}\mathrm{m} 1 & \mathrm{std} 1 & \mathrm{~m} 2 & \mathrm{std} 2 & \mathrm{~m} 3 & \mathrm{std} 3 & \mathrm{~m} 4 & \text { std4}\end{array}\right]$ and consider them as features for image retrieval.

Step 5:- To perform IWT operation consider the following steps

Step a:- On complete gray image apply Integer Wavelet Transform (IWT) using Haar filter. It produce Approximation sub-band. 
Step b:- Generate the Mean (M) and Standard Deviation (Sd) for approximation Sub-band.

Step c:- Above computed feature vectors form as single vector fv3 $=[\mathrm{m} 5 \mathrm{std} 5]$ and consider them as features for image retrieval.

Step 6:- Combine the feature vectors of DWT, SWT \& IWT as single vector. $f v=[f v 1 \mathrm{fv} 2 \mathrm{fv} 3]$ and consider them as features for image retrieval.

Step 7:- Repeat the step 2-6 for currently captured image $\&$ database images. Construct feature vector and store in feature database.

Step 8:- Perform the similarity measurement using Euclidean or city block or minkowski or mehalanobis distance.

Step 9:- Arrange the distances in ascending order.

Step 10:-First $\mathrm{N}$ images considered as resultant images.

\section{ANALYSIS OF EXPERIMENTAL RESULTS}

The table 1 represents the average recognition rate of the query image by measuring the number of images of same category which are found in the top $\mathrm{N}$ matches.

Table 1:- Recognition rate of query image

\begin{tabular}{|l|l|l|l|l|l|l|}
\hline & \multicolumn{7}{|l|}{ No. of top matches } \\
\hline Method & 1 & 2 & 4 & 6 & 8 & 10 \\
\hline HSV histogram & 100 & 88 & 86 & 72 & 62 & 55 \\
\hline DWT & 100 & 95 & 87 & 79 & 69 & 67 \\
\hline SWT & 100 & 94 & 86 & 79 & 69 & 67 \\
\hline DWT \& SWT & 100 & 94 & 88 & 83 & 71 & 70 \\
\hline IWT & 100 & 95 & 89 & 79 & 75 & 71 \\
\hline $\begin{array}{l}\text { DWT, SWT \& } \\
\text { IWT }\end{array}$ & $\mathbf{1 0 0}$ & $\mathbf{9 6}$ & $\mathbf{8 8}$ & $\mathbf{8 2}$ & $\mathbf{7 6}$ & $\mathbf{7 4}$ \\
\hline
\end{tabular}

The recognition rate has been improved by combining the feature vectors of DWT, SWT and IWT methods. The comparison between existing and proposed methods is represented as a graphical form in figure 6 .

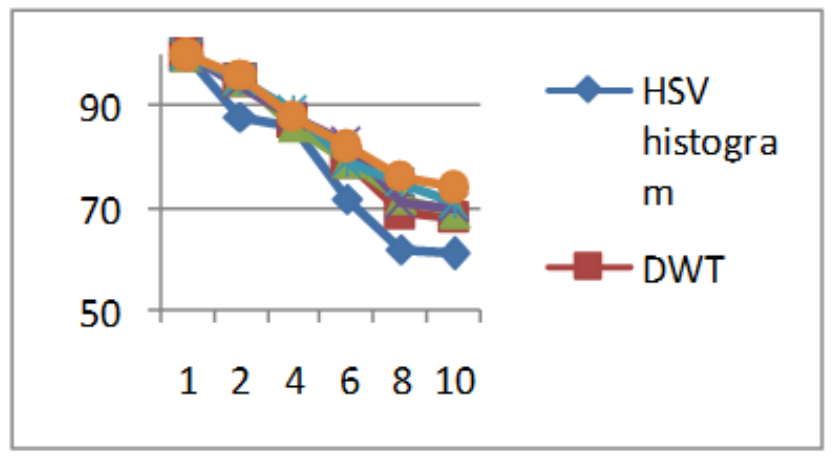

Figure 6 2D Comparative recognition rates.

\section{CONCLUSION}

CBIR using transform domain features has been implemented on COREL database using different distance measures. The proposed algorithm produce $100 \%$ recognition for single image $74 \%$ recognition for top 10 images. Compared to the other existing method the proposed method provides more efficient recognition rate.

\section{REFERENCES}

1. Mohd. Aquid Ansari, Manish Dixit. "An enhanced CBIR using HSV quantization, discrete wavelet transform and edge histogram descriptor", 2017 International Conference on Computing, Communication and Automation (ICCCA), 2017S.

2. Yogita Mistry, D.T. Ingole, M.D. Ingole. "Content based image retrieval using hybrid features and various distance metric", Journal of Electrical Systems and Information Technology, 2017.

3. "CBIR system using color moment and color autocorrelogram with Block Truncation coding". Vandana Vinayak, Sonika Jindal, Computer Science \& Engineering, SBSSTC, Ferozepur D975-8887 Volume 161, March 2017.

4. N. S. Vhang and K. S. Fu, "Query by pictorial example, "IEEE Trans. On Software Engineering, Vol. 6, November, PP 519-524, 1980.

5. Sumaira Muhammad Hayat Khan, Ayyaz Hussain, Imad Fakhri Taha Alshaikhli. "Comparative Study on ContentBased Image Retrieval (CBIR)", 2012 International Conference on Advanced Computer Science Applications and Technologies (ACSAT), 2012.

6. Amit Singla, Meenakshi Garg, "CBIR Approach Based On Combined HSV, Auto Correlogram, Color Moments and Gabor Wavelet", International Journal Of Engineering And Computer Science, 2014.

7. Bin Wang, Sheng Tang, Ruizhen Zhao, Wu Liu, Yigang Cen. "Pedestrian detection based on Region Proposal Fusion", 2015 IEEE 17th International Workshop on Multimedia Signal Processing (MMSP), 2015

8. Madhav, B.T.P, M. Venu Gopal Rao, and V.G.K.M Pisipati. "Multispectral correlations technique for finding phase transition temperatures in 70.Om series", Liquid Crystals Today, 2015.

9. Vipin Tyagi. "Content-Based Image Retrieval", Springer Nature, 2017

10. Kusetogullari, Huseyin, Mark S. Leeson, Burak Kole, and Evor L. Hines. "Meta-heuristic algorithms for optimized network flow wavelet based image coding", Applied Soft Computing, 2013. 